Ann. Biol. anim. Bioch. Biophys., 1979, 19 (1B), 289-302.

\title{
Influence de l'alimentation des truies gravides sur l'évolution des réserves corporelles maternelles et le développement de la portée
}

par M. ETIENNE

Station de Recherches sur l'Elevage des Porcs

I. N. R. A., 78350 jouy en Josas.

Summary. Effect of feeding on pregnant sow body storage and development of the offspring.

The energy and protein nutrition of pregnant sows has a great influence on their weight gain and very little on the progeny ; prolificacy is almost unaffected by maternal nutrient deficiency, and the depression of piglet mean weight at farrowing is limited. The growth rate of the litter between birth and weaning could be affected due to the lower body reserves of the mother, which are utilized in milk synthesis. However, except for extreme feeding conditions like distribution of a protein-free diet during the whole pregnancy, mean daily gain of the piglets is not depressed after weaning. From a qualitative viewpoint, the feeding of the pregnant sow can affect the development of the progeny as well as the composition of maternal weight gain. Pregnancy anabolism, more apparent in sows than in gilts, is essentialiy related to improved protein storage. On the other hand, lipid reserves seem to be more limited and decrease as farrowing approaches, even if dietary energy lallowance is high. Piglet development is more affected than couls be predicted by the limited difference in growth rate and the absence of significant effects at slaughter. The lower piglet weight noticed at birth corresponds to a similar reduction of different organs and tissues. Under energy restriction, liver glycogen storage, important during the first hours of life, is depressed. A drastic reduction of protein or energy allowance to pregnant sows also affects the muscular development of the progeny. This effect seems irreversible, probably because, contrary to rat, muscle fiber differentiation is terminated before birth. Piglet brain development also appears affected by a protein-free diet fed to the pregnant sows.

\section{Introduction.}

Depuis une quinzaine d'années, les recommandations en matière de quantité d'aliment à distribuer aux truies gravides sont passées d'un niveau pléthorique à des apports beaucoup plus limités. Cetłe évolution est logique dans un contexte économique de production au moindre coût, compte tenu des très faibles variations de productivité des femelles malgré des modifications relativement importantes des apports nutritionnels. Cependant, la plupart des expériences ayant permis une felle économie d'aliment se limitent à l'étude des performances globales de reproduction classiquement enregistrées : variations pondérales des truies, nombre et poids des porcelets à la naissance ou à l'issue de la lactation, en relation avec le régime maternel, le but recherché 
étant le sevrage d'un nombre maximal d'animaux à un poids compatible avec de bonnes performances de croissance jusqu'à l'abattage au stade commercial. Il existe comparativement peu de travaux se rapportant plus particulièrement aux modifications de développement du fœetus ou du porcelet nouveau-né en fonction de l'alimentation de la truie, et il paraît intéressant d'en faire le point. Nous nous limiterons aux domaines de la nutrition énergétique et azolée dans lesquels se trouvent le plus d'informations, permettant ainsi leur confrontation. Mais au préalable, il semble opportun d'étudier les effets de l'alimentation pendant la gestation sur l'évolution des réserves corporelles de la truie, compte tenu des relations pouvant exister entre ces dernières ef la croissance des fœtus ou des porcelets allaités.

\section{Alimentation des truies et évolution des réserves corporelles}

\section{Nature des réserves corporelles édifiées par la truie gravide.}

Des travaux déjà anciens de Salmon-Legagneur ef Jacquot (1961a) ou de Heap et Lodge (1967) réalisés sur des truies multipares, ou ceux de Elsley ef al. (1966b) sur femelles nullipares, ont permis de préciser le phénomène d'anabolisme gravidique dans l'espèce porcine : lorsque l'on compare des truies gravides et non gravides de même poids initial, les premières réalisent, outre leur portée, un gain de poids propre qui est supérieur à celui des femelles vides. Ces auteurs soulignent que la majeure partie du gain maternel est fixée dans la carcasse : 94 p. 100 de l'énergie et 75 p. 100 des protéines selon Salmon-Legagneur et Jacquot (1961a) dans le cas d'une alimentation à volonté.

L'augmentation de poids de la truie gravide est assez régulière avec, d'après Lodge et al. (1961), un ralentissement pendant la $6^{\mathrm{e}}$ et après la $12^{\mathrm{e}}$ semaine de gestation. Elle résulte principalement de la prise de poids propre à la mère au début de la gestation, alors que l'utérus gravide se développe essentiellement pendant le dernier tiers (Salmon-Legagneur et Jacquot, 1961a). La nature du gain propre à la truie reste encore imprécise. Lenkeit ef al. (1955) notent que des quantités bien supérieures d'azote peuvent être retenues par la mère elle-même que par les produits de la conception. La rétention azotée augmente entre le début et la fin de la gestation (Lenkeit et al., 1956 ; Salmon-Legagneur, 1965 ; Elsley et al, (1966b). Selon Rombauts (1962), l'anabolisme azoté existe dès les premières semaines qui suivent la saillie, mais l'écart entre truies gravides et non gravides va en s'accentuant surtout en raison de la diminution de rétention azotée chez ces dernières. A cel égard, le comportement des truies nullipares semble particulier : chez les jeunes femelles encore en croissance, qui se trouvent déjà à un niveau d'anabolisme élevó, la gestation ne peut augmenter que faiblement leurs capacités anabolisantes (Rombauts, 1962). Aussi, pour ces animaux, l'amélioration de la rétention azotée avec l'avancement de la gestation n'apparaît pas nettement (Etienne et Henry, 1973).

L'accroissement des dépôts azotés de la truie s'accompagne d'une élévation de la teneur en eau des tissus : Penzes (1959) observe chez les truies gravides un enrichissement en eau de 0,5 à 3 points suivant le tissu ou l'organe considéré. De même, Elsley ef al. (1966b) trouvent une rétention d'eau supérieure chez les femelles gravides, en 
partie dans les tissus maternels, et dans une moindre mesure par une augmentation du volume sanguin.

Si l'augmentation des réserves protéiques des truies pendant la gestation n'est pas discutée, il en va différemment pour les dépôts adipeux. Heap et Lodge (1967) n'observent aucune différence de poids des graisses internes entre les femelles vides ou gravides, mais une quantité de graisses de poitrine plus élevée chez les truies saillies. Inversement, à 30 jours de gestation, Legault et al. (1974) notent des dépôts adipeux moins importants sur la paroi ventrale des truies gravides. A l'approche de la parturition, Elsley et al. (1966b) rapportent que le pourcentage de graisses sous-cutanées est inférieur dans la carcasse des truies qui ont été saillies. A partir d'abattages effectués à différents stades, Salmon-Legagneur et Jacquot $(1961 a, b)$ montrent qu'à un dépôt de lipides au début de la gestation succède leur mobilisation avant la mise bas, qui se traduit par une réduction de 12 p. 100 des graisses sous-cutanées et de 26 p. 100 du gras interne entre le début ef la fin de la gestation. Cette lipolyse, résultant d'une augmentation de la production de chaleur à l'approche de la parturition, est confirmée par Verstegen et al. (1971) par la méthode des échanges respiratoires et par Etienne et Henry (1973) par l'étude de la lipogenèse in vifro. De Wilde ef al. (1974) concluent pour leur part à l'absence d'anabolisme énergétique chez la truie nullipare : la fraction de l'énergie fixée sous forme de graisse dans la carcasse chez les femelles gravides ne représente que 72,1 p. 100 contre 80,5 p. 100 chez les animaux témoins, et le contenu énergétique est lui-même inférieur chez les premières ( 469 contre $492 \mathrm{Mcal}$ ). On peut enfin signaler qu'un certain nombre d'études montrent un amaigrissement des truies entre la saillie et quelques jours après la parturition (Kołarbinska et Kielanowski, 1973; Brooks et al., 1975 ; Hovell ef al., 1977). Mais l'interprétation de ces résultats est difficile, toutes les femelles ayant perdu du poids après la mise bas, même lorsqu'elles n'ont pas allaité.

La gestation se traduit donc chez la truie par un accroissement des dépôts qui pourront être utilisés au cours de la lactation suivante. Cet effet paraît d'autant plus net que la femelle a terminé sa croissance. Au niveau des réserves adipeuses, il semble qu'à un dépôt initial de lipides succède leur mobilisation partielle à l'approche de la parturition.

\section{Influence de l'alimentation sur les réserves de gestation.}

Une élévation de la quantité d'aliment ou de l'apport d'énergie aux truies gravides se traduit par une augmentation marquée du gain de poids total de la femelle, ou de son gain propre. C'est ainsi qu'à partir de calculs effectués sur les données de la bibliographie, Henry et Etienne (1978) trouvent qu'à une augmentation quotidienne de $1000 \mathrm{kcal}$ du niveau d'ingestion d'énergie digestible correspond un accroissement de $8,4 \mathrm{~kg}$ du gain total de gestation, et de 7 à $7,8 \mathrm{~kg}$ du gain net. L'élévation des apports azotés ou l'amélioration de leur qualité à partir d'un niveau suboptimum contribuent également à accroître le gain de poids de gestation de la truie (Duée et Sève, 1978). Mais cet effet est relativement moins important que dans le cas de l'énergie; de plus, la relation entre l'augmentation pondérale des truies et l'élévation du taux azoté de l'aliment semble suivre une évolution curvilinéaire (Kemm et Ras, 1976 ; Greenhalgh et al., 1977), le gain de poids de la femelle n'augmentant plus, dans certaines expérien- 
ces, au-delà d'une consommation journalière de 240 à $290 \mathrm{~g}$ de protéines, pour un niveau des apports d'énergie maintenu constant (Holden et al., 1968 ; Baker et al. 1970 ; Mahan et Mangan, 1975).

En ce qui concerne la nature du gain de poids supplémentaire de la truie, l'augmentation du taux azoté de l'aliment élève la rétention azotée (Rippel ef al., 1965a ; Miller et al., 1969 ; Duée, 1976), mais réduit la fixation d'énergie dans la carcasse (Kemm, 1976). Ainsi, Frape et al. (1971) constatent une diminution d'épaisseur de lard quand l'apport journalier de protéines croît de 207 à $363 \mathrm{~g}$. L'amélioration de l'équjlibre des acides aminés de l'aliment se traduit également par une rétention azotée supérieure, et Rippel ef al. (1965b) basent la détermination du besoin en acides aminés sur l'obtention d'un bilan azoté maximal. L'augmentation de l'apport d'énergie provoque une fixation plus élevée de graisses, cet accroissement restant toutefois moindre que chez des femelles non gravides (Salmon-Legagneur et Jacquot, 1961b ; Frape et al., 1971 ; Hovell ef all., 1977). Elle entraîne de plus, à certains niveaux, une amélioration de la fixation des protéines (Ełienne et Henry, 1973) ; ainsi, SalmonLegagneur (1963) note que le niveau énergétique de la ration constitue davantage un facteur limitant de la synthèse protéique que le niveau azoté lui-même, vraisemblablement en raison d'une augmentation du catabolisme protéique pour les oxydations cellulaires dans le cas d'une insuffisance en énergie (Ruiz ef al., 1971). On peuł d'ailleurs remarquer que l'augmentation de la rétention azotée en fin de gestation n'apparaît pas chez la truie nullipare lors d'un apport énergétique suboptimal (Etienne et Henry, 1973) ou d'une déficience en certains acides aminés, tel la thréonine (Duée, 1977).

La nature des réserves édifiées pendant la gestion peut donc être modulée par le biais de l'alimentation ; mais à cet égard, la truie gravide présente un comportement particulier puisqu'elle paraît avoir des potentialités de dépôt de tissu maigre supérieures et de tissu gras moindres que le porc adulte.

\section{Alimentation de la truie gravide et développement des porcelets}

L'alimentation de la truie gravide est susceptible d'influencer le développement des porcelets de plusieurs façons : soit directement au niveau de la portée in utero, soit indirectement sur la croissance du porcelet nouveau-né par le biais du lait produit, dans la synthèse duquel interviennent en partie les réserves corporelles édifiées par la mère au cours de la gestation précédente. Enfin, on peut se demander si les effets constatés sur le porcelet nouveau-né ou sevré persistent jusqu'à un stade ultérieur du développement de l'animal.

1. Alimentation de la truie gravide ef développement fœtal.

Si l'on en juge d'après le poids du porcelet à la parturition, l'alimentation de la truie gravide affecte la croissance fœetale : une réduction des apports énergétiques (Henry ef Etienne, 1978) ou azotés (Duée et Sève, 1978), ou même une carence en lysine (Duée et Rérat, 1975), diminue le poids moyen des porcelets à la naissance. Cet effet est d'autant plus marqué que la restriction est plus sévère, ou appliquée de façon plus durable, des effets cumulatifs apparaissant au fur et à mesure des cycles successifs. 
Ainsi, pour chaque apporł supplémentaire de $1000 \mathrm{kcal}$ d'énergie digestible par jour à la truie gravide, le porcelet nouveau-né pèse en moyenne $15 \mathrm{~g}$ de plus au premier cycle de reproduction $(r=0,30)$, ef $37 \mathrm{~g}$ de plus $(r=0,65)$ quand la même addition est faite pendant au moins deux gestations (Henry et Etienne 1978). Mais l'influence de la nutrition maternelle sur le poids du porcelet à la naissance reste limitée en regard des effects sur le gain maternel. En outre, la taille de la portée n'est que rarement affectée : la fonction de reproduction est assurée de façon prioritaire par rapport à la fonction de croissance du fœetus, et surtout de la mère.

En ce qui concerne la croissance fœtale proprement dite, le jeûne de la truie à partir de 10 jours avant la saillie entraîne une réduction de la longueur des fœtus, du poids ef du contenu azoté des fotus et de leurs annexes à 30 jours de gestation, sans que la mortalité embryonnaire soit affectée (Anderson, 1975) ; mais la gestation ne peut se poursuivre dans ces conditions extrêmes au-delà de 34 jours, les truies avortant. Une restriction énergétique ou azotée moins sévère ne provoque aucun effet significatif sur le poids des fœtus à 30 jours (Heap et al., 1967 ; Etienne et Duée, 1973) ni à 60 ou 70 jours de gestation (Pike et Boaz, 1972 ; Leuillet et al., 1979) sans doute parce que, ce n'est qu'après ce stade que la croissance pondérale des embryons est rapide, et donc plus exigeante en nutriments.

Une diminution importante des apports d'énergie dans l'alimentation des truies gravides provoque une réduction pondérale de différents organes : le poids du foie (Anderson et Wahlstrom, 1970), des reins, du pancréas, des surrénales, du muscle gastrocnémien est abaissé dans la même proportion que le poids du porcelet, alors que cet effet est moindre pour la thyroïde, et nul pour le cerveau (Buitrago ef al., 1974a ; tabl. 1). Parallèlement, la concentration ou la quantité totale d'ADN, le rapport

\section{TABLEAU 1}

Influence de l'apport d'énergie en gestation sur le développement des porcelets à la naissance

(Buitrago ef al., 1974) (1)

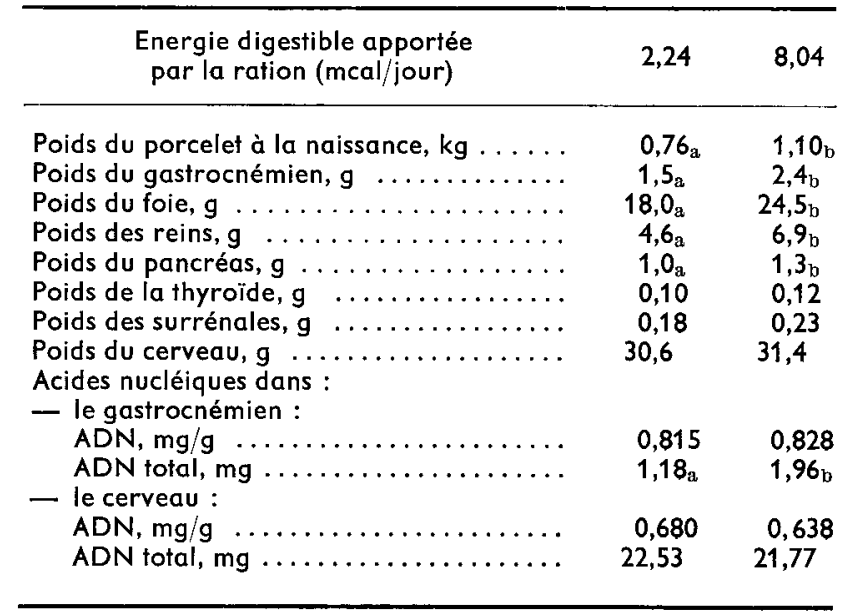

(1) Les valeurs d'une même ligne indexées par des lettres différentes sont significativement différentes $(P<0,05)$. 
ADN/protéines dans le cerveau (Buitrago ef al., 1974a) ef son contenu en ARN (Atinmo et al., 1974b) restent inchangés, tandis que la quantité d'ADN dans le gastrocnémien est significativement plus faible à la naissance à la suite d'une carence sévère et prolongée en énergie de la truie gravide (Buitrago et al., 1974a ; tabl. 1). La glycémie (Seerley et al., 1974), le taux de protéines et d'albumine sériques ou d'hémoglobine du sang des porcelets sont abaissés (Atinmo et al., 1974b), ainsi que la teneur en lipides du muscle longissimus dorsi (Buitrago et al., 1974a). Cependant, pour des carences moins marquées de la mère, on ne peut mettre en évidence aucune différence de composition chimique globale de la carcasse des porcelets (Etienne et Henry, 1973 ; Elliot ef Lodge, 1977 ; Okai ef al., 1977).

Les effets d'un supplément d'énergie fourni après 100 jours de gestation ont été recherchés par l'addition de glucides ou de lipides au régime de base de la truie L'adjonction d'huile de mais provoque un enrichissement des lipides du foie et du muscle des porcelets en acide linoléique, ce qui laisse penser à un passage transplacentaire de cet acide gras en fin de gestation (Seerley et al., 1974). La teneur en glycogène du foie est augmentée (Elliot et Lodge, 1977 ; Seerley et al., 1974), pouvant passer de 11,7 à 14,5 p. 100 , mais à apport énergétique égal, la supplémentation en glucides paraît plus efficace qu'en lipides à cel égard (Okai et al., 1978). Le même effet est parfois constaté dans le muscle (Anderson et Wahlstrom, 1970 ; Seerley et al., 1974) ; les écarts restent cependant faibles, voire non significatifs (Elliot et Lodge, 1977). Cette différence observée entre les localisations peut s'expliquer par l'augmentation tardive des dépôts de glycogène, ef leur plus grande concentration dans le foie que dans le muscle.

Les effets d'un régime presque totalement dépourvu de protéines $(0,5$ à 3 p. 100 de l'aliment) sur le développement des fœus ont été recherchés dans plusieurs expériences. Si la reproduction est possible dans ces conditions extrêmes, le poids à la naissance des porcelets est en général abaissé. La distribution d'un régime normal au

TABLEAU 2

Influence d'une restriction énergétique ou azotée pendant la gestation sur le développement des porcelefs ò la naissance (Atinmo ef al., 1974b)

\begin{tabular}{|c|c|c|c|}
\hline Lot & Témoin & $\begin{array}{l}\text { Restreint } \\
\text { en énergie }\end{array}$ & $\begin{array}{l}\text { Restreint } \\
\text { en protéines }\end{array}$ \\
\hline Niveau d'alimentation, $\mathrm{kg} / \mathrm{j}$ & 1,82 & 0,91 & 1,82 \\
\hline Protéines ingérées, $g / j \ldots \ldots$ & 323 & 323 & \\
\hline Poids du porcelet à la naissance, $\mathrm{kg}$ & $1,29_{\mathrm{a}}$ & $1,13_{\mathrm{a}}$ & $0,98_{\mathrm{b}}$ \\
\hline Poids du foie, $g \ldots \ldots \ldots \ldots \ldots$ & $36,2_{a}$ & $28,9 \mathrm{a}$ & $16,7_{\mathrm{b}}$ \\
\hline Poids des reins, $g$ & 9,4 & 7,2 & 8,2 \\
\hline Poids des surrénales, $g \ldots \ldots \ldots \ldots$ & 0,27 & 0,24 & 0,23 \\
\hline Poids du cerveau, g $\quad \ldots \ldots \ldots \ldots$ & $33,0_{\mathrm{a}}$ & $33,1_{\mathrm{a}}$ & $31,1_{b}$ \\
\hline \multicolumn{4}{|l|}{ - le muscle longissimus dorsi : } \\
\hline $\begin{array}{l}\text { Ie muscle iongissimus dorsi : } \\
\text { ADN, } \mathrm{mg} / \mathrm{g} \ldots \ldots \ldots \ldots \ldots\end{array}$ & 2,07 & 1,59 & 1,61 \\
\hline \multirow{4}{*}{ 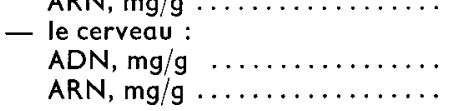 } & 1,71 & 1,28 & 1,28 \\
\hline & & & \\
\hline & 0,87 & 0,76 & 0,85 \\
\hline & $1,39 \mathrm{a}$ & $1,00_{\mathrm{a}}$ & $0,90_{b}$ \\
\hline
\end{tabular}


moment de l'implantation des blastocytes permet de pallier partiellement cet effet (Pond ef al., 1969) ; en fait, l'apport transitoire de protéines per se interviendrait plus que le moment auquel il est effectué (Leuillef et al., 1979). Le poids de différents organes et tissus des porcelets est diminué à la naissance (Frape et al., 1969 ; Leuillet et al., 1979) comme pour une carence en énergie, mais le développement du cerveau paraît également atteint : si sa teneur en ADN est inchangée, son poids et la concentration en ARN sont abaissés lorsque les mères ingèrent $9 \mathrm{~g}$ de protéines par jour (Atinmo et al., $1974 b$; tabl. 2). Le développement musculaire est affecté, la teneur en protéines de la carcasse étant d'autant plus faible que la carence azotée est sévère (Elsley et al., 1966a ; Leuillet et al., 1979). Des effets ont même pu être constatés pour des apports relafivement importants : selon Livingston (1962), les porcelets à la naissance auraient des fibres musculaires de diamètre significativement supérieur de 3,66 et 3,23 $\mu$ respectivement dans les muscles longissimus dorsi et psoas majoris lorsque les mères ingèrent quotidiennement 520 au lieu de $257 \mathrm{~g}$ de protéines; cet écart n'est cependant pas retrouvé par la suite. Au niveau sanguin, une carence azotée sévère diminue le taux de protéines et d'albumine sériques des fœtus âgés de 13 semaines (Atinmo et al., 1976a) ou à la naissance (Atinmo et al., 1974b). Le même effet est constaté plus précocément chez les mères gravides et évoque un épuisement de leurs réserves labiles, (Atinmo ef al., 1974a ; Pond ef al., 1968, 1969).

Le niveau plasmatique de certaines hormones a été déterminé dans des conditions nutritionnelles analogues à partir de 10 semaines de gestation. Le taux de GH maternelle ou fœtale n'est pas influencé par la nutrition azotée de la truie (Atinmo et al., 1976b). Il est très élevé chez les fœtus, mais ne semble pas avoir de signification avant la naissance, compte tenu de l'absence de relation entre le niveau de $\mathrm{GH}$ et la croissance embryonnaire. Après la naissance, le taux de $\mathrm{GH}$ plasmatique des porcelets diminue, sauf chez ceux qui sont issus de truies très carencées en protéines, bien qu'ils soient allaités par des femelles témoins. Ceci pourrait indiquer une altération du fonctionnement de l'hypophyse chez ces animaux, ou une mauvaise utilisation de la $\mathrm{GH}$, si tant est que le niveau sanguin de cette hormone puisse être relié à des différences de croissance des animaux.

L'insulinémie des fœtus augmente d'autant plus après 13 semaines de gestation que la mère ingère davantage de protéines (Atinmo et al., 1976c) ; la même différence est observée plus tôt chez la truie. Cette hormone aurait un rôle anabolique durant la vie fœtale, une corrélation élevée existant entre l'insulinémie et la croissance des fœutus $(r=0,84)$. Le taux d'insuline plasmatique mesuré jusqu'à 10 semaines d'âge reste plus faible chez les porcelets dont les mères ont consommé un régime presque totalement dépourvu de protéines, bien qu'ils soient allaités par des truies ingérant un régime témoin, puis reçoivent un aliment équilibré. Mais on ne peut savoir si cet écart est dû à une lésion permanente de fonctionnement du pancréas de ces animaux.

\section{Alimentation de la truie gravide et développement des porcelets allaités.}

La nutrition énergétique de la truie gravide n'affecte pas en général la survie postnatale des porcelets. Seule l'étude de Seerley et al. (1974) conclut à une mortalité moindre des jeunes issus de truies ayant consommé un supplément d'huile de maïs, peut-être à cause des réserves de glycogène plus importantes des porcelets à la nais- 
sance. Cependant, celles-ci sont rapidement dissipées, 75 p. 100 du glycogène hépatique ayant disparu $12 \mathrm{~h}$ après la naissance (Elliot et Lodge, 1977), et les différences constatées à la mise bas ne persistent pas; ceci expliquerait que dans le travail de Seerley et al. (1974), la thermostabilité des porcelets mesurée à 6 et $54 \mathrm{~h}$ d'âge en les soumettant durant $90 \mathrm{~mm}$ à une température de $3^{\circ} \mathrm{C}$ ne diffère pas significativement entre les traitements. La vitesse de croissance des porcelets s'élève avec le niveau de consommation de la truie en gestation, mais il semble qu'il faille davantage d'aliment pour rendre maximum le poids au sevrage qu'à la naissance (Baker et al., 1969).

La réduction de la quantité de protéines ingérées par la truie en gestation provoque souvent une diminution de la viabilité des porcelets jusqu'au sevrage, même pour des apports assurant des performances de mise bas normales, tels des régimes constitués pour plus de 96 p. 100 de céréales : orge (Young ef al., 1976) ou maïs (Baker et al., 1970). La vitesse de croissance des porcelets tend à diminuer. Cet effet paraît d'autant plus marqué que la taille de la portée est importante (Young et al., 1976) ou que le niveau de consommation de protéines de la truie est faible pendant la lactation (Mahan et Mangan, 1975).

Pour quelles raisons la croissance des porcelets est-elle déprimée à la suite d'une restriction de la consommation des nutriments énergétiques ou azotés par la mère en gestation ? Selon Henry et Etienne (1978), cet effet serait en partie lié aux différences de poids des jeunes enregistrées à la naissance. Les modifications de développement des individus pourraient intervenir, mais ceci n'a pas été bien établi. L'écart initial se trouverait accentué par la têtée plus rapide des porcelets vigoureux (England, 1974) et, de façon paradoxale, par la tendance parfois constatée pour les portées les plus lourdes à la naissance à consommer davantage d'aliment sec avant le sevrage (O'Grady, 1967 ; Nielsen, 1970). Une autre cause importante réside dans l'influence de l'alimentation durant la gestation sur la production laitière, par le biais des réserves corporelles constituées par la truie avant la parturition. Ce phénomène a été bien démontré lors de carences azotées sévères en gestation, par échange de porcelets entre les mères à la mise bas : la vitesse de croissance des porcelets dépend du régime de gestation de la truie qui les allaite, et non de celui qu'avait reçu la femelle dont ils sont issus (Pond et al., 1968 ; De Geeter ef al., 1972 ; Hammell et al., 1976).

L'intervention des réserves corporelles dans la synthèse lactée apparaît de façon globale dans la relation étroite qui existe entre le gain de poids des mères pendant la gestation ef leur perte pondérale au cours de la lactation suivante. De même, au plan métabolique, l'incorporation d'huile de maïs dans le régime de la truie gravide élève le taux d'acide linoléique dans le tissu adipeux maternel avant la parturition, puis dans les lipides du lait (Salmon-Legagneur, 1964). Ceci expliquerait qu'un accroissement des apports énergétiques à la femelle en gestation provoque une augmentation de la production laitière (Salmon-Legagneur et al., 1960), du taux de lipides du colostrum (Seerley et al., 1974) et du lait, élevant ainsi sa teneur en énergie (Van Spaendonck, 1972). La distribution d'un régime à teneur plus faible en protéines avant la mise bas contribue parfois à diminuer la teneur en matières azotées du colostrum (Elliott et al., 1971) ou du lait (Holden et al., 1968). De cet ensemble de facteurs liés à la nutrition maternelle pendant la gestation pourraient résulter les différences de viabilité et de croissance des porcelets allaités. Mais en réalité, des effets à ce niveau dépendentégale- 
ment de l'alimentation de la truie durant la lactation, et des interactions entre les apports nutritionnels avant ef après la parturition.

\section{Alimentation de la truie gravide ef développement des porcelets sevrés.}

Les effets éventuels du régime maternel durant la gestation sur la croissance des porcelets entre le sevrage et l'abattage effectué à un poids vif de 90 à $100 \mathrm{~kg}$ ont parfois été recherchés. Pour des niveaux de rationnement énergétique variés (Atinmo et al., $1974 b$; Buitrago et al., $1974 b$; Gondos ef al., 1975), pour des apports de l'ordre de 150 à $180 \mathrm{~g}$ de protéines par jour (Livingstone ef al., 1966 ; Kemm et Pieterse, 1968 ; Hawton et Meade, 1971) ou dans le cas d'une carence en lysine du régime de gestation (Duée et Rérat, 1975), la vitesse de croissance des animaux sevrés n'est pas affectée de façon significative. La différence de poids constatée au sevrage se trouve ainsi maintenue, ou faiblement accentuée. Ainsi, Vermedahl et al. (1969) observent un écart de 1,7 jour pour que des porcs issus de truies ayant consommé 1,36 ou 2,27 kg d'aliment/jour atteignent le poids de $91 \mathrm{~kg}$. Au contraire, dans les essais comportant la distribution de régimes protéiprives pendant la gestation, l'effel dépressif noté sur la croissance des porcelets jusqu'au sevrage persiste en général par la suite (De Geeter et al., 1973 ; Atinmo et al., 1974b) ; la diminution du gain moyen quotidien est d'autant plus importante que le régime dépourvu de protéines a été distribué plus longtemps à la mère (Pond et al., 1969). Mais dans aucune de ces expériences, l'efficacité alimentaire n'est affectée.

Au niveau de la composition corporelle des porcs à l'abattage, le muscle longissimus dorsi a très souvent été retenu comme estimateur du développement musculaire. Dans aucune expérience, la surface de sa section n'est influencée par la nature ou par l'ampleur de la restriction nutritionnelle imposée à la mère gravide. De même, les pourcentages de jambon ef de longe dans la carcasse restenf inchangés (Vermedahl ef al., 1969 ; Hawton et Meade, 1971). Cependant, il a parfois été trouvé que la teneur en lipides du muscle des porcs à l'abattage avait tendance à augmenter à la suite d'un rationnement énergétique (Vermedahl et al., 1969) ou azoté de la mère (Hawton et Meade, 1971 ; Haught ef al., 1977). Ce fait est à rapprocher d'un accroissement de l'épaisseur du lard dorsal des individus dans les mêmes conditions (Vermedahl ef al., 1971 ; Kemm et Pieterse, 1968 ; Haught et al., 1977). Selon Robinson (1969), la distribution quotidienne de $0,9 \mathrm{~kg}$ d'aliment à la truie gravide provoque un arrêt de l'augmentation du contenu en ADN du muscle à 60 jours d'âge des porcelets, au lieu de 80 à 100 jours dans le cas d'un apport de $1,8 \mathrm{~kg} / \mathrm{jour}$; les animaux ayant subi un stress au début de leur développement auraient tendance à déposer des lipides plus précocément et, pour un poids à l'abattage identique, présenteraient une plus grande proportion de graisses intramusculaires. Cette remarque rejoint les observations de Allen et al. (1974) selon lesquelles les porcelets les plus chétifs à la naissance auraient des fibres musculaires de diamètre supérieur, mais en nombre moindre que leurs frères de portée normaux ; ils seraient donc physiologiquement plus matures du point de vue de leur croissance musculaire. Des différences analogues de développement musculaire ont été relevées par Pond et al. (1969), le rapport ARN/ADN dans le muscle long dorsal étant réduit chez les porcs de $90 \mathrm{~kg}$ issus de truies soumises à un régime protéiprive en gestation, ce qui pourrait signifier une activité de synthèse de protéines dans le 
muscle moindre chez ces animaux ; ce fait n'est cependant pas retrouvé par Atinmo et al. (1974b).

Les effets constatés sur le cerveau du porcelet nouveau-né persistent à $90 \mathrm{~kg}$ de poids vif : la concentration ef la quantité d'ADN présentes dans le cerveau ou le cervelet sont inchangées, alors qu'elles sont abaissées pour l'ARN lorsque la mère gravide a reçu un régime presque totalement dépourvu de protéines (Pond et al., 1969 ; Atinmo et al., 1974b). Ces différences biochimiques sont à rapprocher des études de comportement effectuées par Hammell et al. (1976) sur des porcelets placés dans des labyrinthes à 21 et 45 jours d'âge. Dans l'un de ces essais, le nombre d'erreurs commises par les porcelets et le temps qu'ils mettent pour sortir du labyrinthe sont significativement supérieurs lorsque la truie dont ils sont issus a été carencée en protéines en gestation ef en lactation.

\section{Conclusion.}

Si l'on raisonne au seul point de vue pondéral, la nutrition énergétique et azotée de la truie gravide influence de façon importante son propre gain corporel, et relativement peu sa portée. Une carence, même sévère en gestation a peu ou pas d'effet sur la prolificité, et un effet limité sur le poids des porcelets à la naissance. Leur croissance ultérieure peut être ralentie jusqu'au sevrage, en raison justement de la réduction des réserves tissulaires maternelles qui sont utilisées pour la synthèse du lait. Mais par la suite, cette influence néfaste ne persiste plus jusqu'au poids d'abattage. Seules des conditions nutritionnelles extrêmes, telles celles causées par la distribution d'un régime presque totalement dépourvu de protéines, affectent la vitesse de croissance des animaux au-delà du sevrage.

Quand on s'attache à savoir ce que recouvrent sur le plan qualitatif les différences de croissance observées en fonction du régime de la truie gravide, des particularités apparaissent au niveau du développement de la progéniture aussi bien que de la composition du gain de poids maternel. En ce qui concerne la truie, l'anabolisme gravidique, apparent surtout chez la femelle multipare, correspond en premier lieu à une amélioration du dépôt de protéines par rapport à l'animal non gravide, alors que la mise en réserve de lipides paraît plus limitée, et décroît à l'approche de la parturition, même dans le cas d'une alimentation énergétique libérale. Quant aux porcelets, l'influence de la nutrition de la mère en gestation affecte davantage leur développement que ne le laissent supposer les modifications limitées de vitesse de croissance, ou l'absence de différences notables à la découpe des animaux à l'abattage. La réduction pondérale observée à la naissance se traduit par une diminution de poids de différents organes, sans que l'on sache si leur fonction est lésée. Dans le cas d'une carence en énergie, les réserves de glycogène, importantes au cours des premières heures de la vie des animaux, sont diminuées. Une restriction sévère en protéines ou en énergie de la truie gravide affecte aussi le développement musculaire ; cet effet semble irréversible, sans doute parce qu'au contraire du rat, la différenciation des fibres musculaires est achevée avant la naissance. La distribution d'un régime protéiprive à la mère semble également atteindre de façon irrémédiable le développement du cerveau. 


\section{Références}

ALLEN C. E., THOMPSON E. H., HEGARTY P. V. J., 1974. Physiological maturity of muscle and adipose cells in meat animals. Proc. 27th ann. Reciprocal Meat Conf. amer. Meat Sci. Assoc., 8-27.

ANDERSON L. L., 1975. Embryonic and placental development during prolonged inanition in the pig. Am. J. Physiol., 229, 1687-1694.

ANDERSON R. H.,WAHLSTROM R. C., 1970. Effects of energy intake and dichlorvos during gestation on reproductive performance of gilts and some chemical characteristics of the offspring. J. anim. Sci., 31, 907-916.

ATINMO T., POND W. G., BARNES R. H., 1974a. Effect of dietary energy vs. protein restriction on blood constifuents and reproductive performance in swine. J. Nutr., 104, 1033-1040.

ATINMO T., POND W. G., BARNES R. H., 1974b. Effect of maternal energy vs. protein restriction on growth and development of progeny in swine. J. anim. Sci., 39, 703-711.

ATINMO T., BALDIJAO C., POND W. G., BARNES R. H., 1976a. Prenatal and postnatal protein malnutrition in pigs : effects on growth rate, serum protein and albumin. J. anim. Sci., 43, 606612.

ATINMO T., BALDIJAO C., POND W. G., BARNES R. H., 1976b. Decreased dietary protein or energy intake and plasma growth hormone levels of the pregnant pig, its fetuses and developing progeny. J. Nutr., 106, 940-946.

ATINMO T., BALDIJAO C., POND W. G., BARNES R. H., 1976c. Maternal protein malnutrition during gestation alone and its effects on plasma insulin levels of the pregnant pig, its fetuses and the developing offspring. J. Nutr., 106, 1647-1653.

BAKER D. H., BECKER D. E., NORTON H. W., SASSE C. E., JENSEN A. H., HARMON B. G., 1969. Reproductive performance and progeny development in swine as influenced by feed intake during pregnancy. J. Nutr., 97, 489-495.

BAKER D. H., BECKER D. E., JENSEN A. H., HARMON B. G., 1970. Protein source and level for pregnant gilts : a comparison of corn, opaque 2 corn and corn-soybean meal diets. J. anim. Sci., 30, 364-367.

BROOKS P. H., COLE D. J. A., JENNINGS W. J. N., 1975. Meat production from pigs which have farrowed. 2. Carcass characteristics. Anim. Prod., 20, 123-131.

BUITRAGO J. A., WALKER Jr E. F., SNYDER W. I., POND W. G., 1974a. Blood and tissue traits in pigs at birth and at 3 weeks from gilts fed low or high energy diets during gestation. J. anim. Sci., 38, 766-771.

BUITRAGO J. A., MANER J. H., GALLO J. T., POND W. G., 1974b. Effect of dietary energy in gestation on reproductive performance of gilts. J. anim. Sci., 39, 47-52.

DE GEETER M. J., HAYS V. W., KRATZER D. D., CROMWELL G. L., 1972. Reproductive performance of gilts fed diets low in protein during gestation and lactation. J. anim. Sci., 35, 772-777.

DE GEETER M. J., HAYS V. W., KRATZER D. D., CROMWELL G. L., 1973. Postweaning performance of pigs from gilts fed diets high and low in protein during gestation and lactation. $J$. anim. Sci., 37, 459-462.

DE WILDE R., VAN SPAENDONCK R., VANSCHOUBROEK F., 1974. Energy retention of pregnant and non pregnant gilts, 197-200. In MENKE K. H., LANTZCH H. J., REICHL J. R., Energy melabolism of farm animals, EAAP Pub. $n^{\circ} 14$, Univ. Hohenheim, Stuttgart.

DUÉE P. H., 1976. Chronologie de l'apport azoté pendant le cycle de reproduction chez la truie. Ann. Zootech., 25, 199-212.

DUÉE P. H., 1977. Threonine requirements of pregnant gilts. Proc. 5th int. Symp. Amino Acids, Budapest.

DUÉE P. H., RÉRAT A., 1975. Ełude du besoin en lysine de la truie gestante nullipare. Ann. Zootech., 24, 447-464.

DUÉE P. H., SÈVE B., 1978. Alimentation azotée du porc. Journées Rech. Porcine en France, I. N. R. A.I. T. P. éd., Paris, 10, 167-207.

ELLIOT J. I., LODGE G. A., 1977. Body composition and glycogen reserves in the neonatal pig during the first 96 hours postpartum. Can. J. anim. Sci., 57, 141-150.

ELLIOTT R. F., VANDER NOOT G. W., GILBREATH R. L., FISHER H., 1971. Effect of diefary protein level on composition changes in sow colostrum and milk. J. anim. Sci., 32, 1128-1137. 
ELSLEY F. W. H., MCDONALD I., MAC PHERSON R. M., 1966a. The effect of feed intake and protein concentration during pregnancy on the nitrogen content of newly born pigs. Anim. Prod., 8, 353 (abstr.).

ELSLEY F. W. H., ANDERSON D. M., MCDONALD I., MAC PHERSON R. M., SMART R., 1966b. A comparison of the live-weight changes, nitrogen retention and carcass composition of pregnant and non-pregnant gilts. Anim. Prod., 8, 391-400.

ENGLAND D. C., 1974. Husbandry components in prenatal and perinatal development in swine. J. anim. Sci., 38, 1045-1049.

ETIENNE M., DUÉE P. H., 1973. Effets respectifs des niveaux alimentaires pendant la croissance et le premier mois de gestation sur les performances de reproduction chez la truie nullipare : résultats préliminaires. Ann. Zootech., 22, 453-462.

ETIENNE M., HENRY Y., 1973. Influence de l'apport énergétique sur l'utilisation digestive et métabolique des nutriments, et les performances de reproduction chez la truie gestante nullipare. Ann. Zootech., 22, 311-326.

FRAPE D. L., WOLF K. L., WILKINSON J., CHUBB L. G., 1969. Liver weight and its N and vitamin A contents in piglets from sows fed two levels of protein and food. J. agric. Sci., 73, 33-40.

FRAPE D. L., WILKINSON J., CHUBB L. G., WOLF K. L., 1971. The response of breeding sows and gilts to two levels of protein and food intake over several parities. J. agric. Sci., 76, 73-82.

GONDOS M., HARSIAN E., HARSIAN A., 1975. Recherches concernant les performances à l'engraissement des produits obtenus des truies nourries de manière différente pendant la gestation (roumain). Lucr. Stiint. Inst. Cerc. Pentru Nutr. Anim., 4, 131-144.

GREENHALGH J. F. D., ELSLEY F. W. H., GRUBB D. A., LIGHTFOOT A. L., SAUL D. W., SMITH P., WALKER N., WILLIAMS D., YEO M. L., 1977. Coordinated trials on the protein requirements of sows. 1. A comparison of four levels of dietary protein in gestation and two in lactation. Anim. Prod., 24, 307-321.

HAMMELL D. L., KRATZER D. D., CROMWELL G. L., HAYS V. W., 1976. Effect of protein malnutrition of the sow on reproductive performance and on postnatal learning and performance of the offspring. J. anim. Sci., 43, 589-597.

HAUGHT D. G., TANKSLEY T. D., HESBY J. H. Jr., GREGG E. J., 1977. Effect of protein level, protein restriction and cottonseed meal in sorghum-based diets on swine reproductive performance and progeny development. J. anim. Sci., 44, 249-256.

HAWTON J. D., MEADE R. J., 1971. Influence of quantity and quality of protein fed the gravid female on reproductive performance and development of offspring in swine. J. anim. Sci., 32, 88-95.

HEAP F. C., LODGE G. A., 1967. Changes in body composition of the sow during pregnancy. Anim. Prod., 9, 237-245.

HEAP F. C., LODGE G. A., LAMMING G. E., 1967. The influence of plane of nutrition in early pregnancy on the survival and development of embryos in the sow. J. Reprod. Fert., 13, 269-280.

HENRY Y., ETIENNE M., 1978. Alimentation énergétique du porc. Journées Rech. Porcine en France, INRA-ITP éd., Paris, 10, 119-165.

HOLDEN P. J., LUCAS E. W., SPEER V. C., HAYS V. W., 1968. Effect of protein level during pregnancy and lactation on reproductive performance in swine. J. anim. Sci., 27, 1587-1590.

HOVELL F. D. De B., MAC PHERSON R. M., CROFTS R. M. J., SMART R. I., 1977. The effect of pregnancy, energy intake and mating weight on protein deposition and energy retention of female pigs. Anim. Prod., 25, 281-290.

KEMM E. H., 1976. The protein and energy requirements of the pregnant gilt. 3. The efficiency with which dietary protein and energy are utilized. Agroanimalia, 8, 141-146.

KEMM E. H., PIETERSE P. J. S., 1968. The influence of protein level on the productivity of Large White gilts. Proc. S. Afr. Soc. Anim. Prod., 7, 133-135.

KEMM E. H., RAS M. N., 1976. The protein and energy requirements of the pregnant gilt. 1. Live mass gain. Agroanimalia, 8, 123-130.

KOTARBINSKA M., KIELANOWSKI J., 1973. A note on meat production from pigs slaughtered after first weaning a litter. Anim. Prod., 17, 317-320.

LEGAULT C., GRUAND J., GONDOUIN R., 1974. Note sur l'évolution de l'épaisseur du lard dorsal et de la composition corporelle des jeunes femelles entre 90 et $169 \mathrm{~kg}$ de poids vif : incidence de la gestation. Journées Rech. Porcine en France, INRA-ITP éd., Paris, 6, 257-265. 
LENKEIT W., GÜTTE J. O., WARNECKE W., KIRCHHOFF W., $1955 . \quad$ Langfristige Unfersuchungen zum aüsseren und inneren Stoffwechsel des graviden und lakłierenden Schweines. 3. Mitteilung : Die Beziehung der N-Retention während der Gravidität zum N Umsatz nach der Geburt bei hoher Milchleistung und bei verhinderter Laktation. Z. Tierernähr. Futtermittelkde, 10, 351-364.

LENKEIT W., GÜTTE J. O., KIRCHHOFF W., SOEHNGEN F. K., FARRIES E., 1956. Weitere Untersuchungen zur Abhängigkeit des $\mathrm{N}$-Umsatzes während der Laktation von der Nährstoffversorgung während der Gravidität. 4. Mitteilung der «Langfristigen Untersuchungen zum aüsseren und inneren Stoffwechsel des graviden und laktierenden Schweines ». Z. Tierernähr. Futfermittelkde, 11, 337-352.

LEUILLET M., ETIENNE M., SALMON-LEGAGNEUR E., 1979. Influence d'une très forte restriction azotée à différentes périodes de la gestation de la truie sur le développement des fœłus. Ann. Biol. anim. Bioch. Biophys., 19, 217-223.

LIVINGSTON D. M. S., 1962. The effect of protein nutrition of the pregnant sow on the muscle fibre diameter of the young pig. Anim. Prod., 4, 296 (abstr.).

LINVINGSTONE R. M., MAC PHERSON R. M., ELSLEY F. W. H., LUCAS I. A. M., LODGE G. A., 1966. A note on the effect of protein concentration in the diefs of pregnant sows on the performance and carcass quality of their progeny. Anim. Prod., 8, 337-339.

LODGE G. A., MCDONALD I., MAC PHERSON R. M., 1961. Weight changes in sows during pregnancy and lactation. Anim. Prod., 3, 269-275.

MAHAN D. C., MANGAN L. T., 1975. Evaluation of various protein sequences on the nutritional carry-over from gestation to lactation with first-litter sows. J. Nutr., 105, 1291-1298.

MILLER G. M., BECKER D. E., JENSEN A. H., HARMON B. G., NORTON H. W., 1969. Effect of protein intake on nitrogen retention by swine during late pregnancy. J. anim. Sci., 28, 204-207.

NIELSEN H. E., 1970. Recent danish investigations on the nutrition of sows. Proc. RITENA, Mallorca, $101-116$.

O'GRADY J. F., 1967. Effect of level and pattern of feeding during pregnancy on weight change and reproductive performance of sows. Ir. J. agric. Res., 6, 57-71.

OKAI D. B., AHERNE F. X., HARDIN R. T., 1977. Effects of sow nutrition in late gestation on the body composition and survival of the neonatal pig. Can. J. anim. Sci., 57, 439-448.

OKAI D. B., WYLLIE D., AHERNE F. X., EWAN R. C., 1978. Glycogen reserves in the fetal and newborn pig. J. anim. Sci., 46, 391-401.

PENZES L., 1959. Die Untersuchung des Eiweisseinbaus an Schweinen während der Trächtigkeif (hongr.). Allatienyesztes, 8, 243-246.

PIKE I. H., BOAZ T. G., 1972. The effect of condition at service and plane of nutrition in early pregnancy in the sow. I. Uterine and extra-uterine changes. Anim. Prod., 15, 147-155.

PONDW. G., WAGNER W. C., DUNN J. A., WALKER E. F. Jr., 1968. Reproduction and early postnatal growth of progeny in swine fed a protein-free dief during gestation. J. Nufr., 94, 309-316.

POND W. G., STRACHAN D. N., SINHA Y. N., WALKER E. F. Jr., DUNN J. A., BARNES R. H., 1969. Effect of protein deprivation of swine during all or part of gestation on birth weight, postnatal growth rate and nucleic acid content of brain and muscle of progeny. J. Nutr., 99, 61-67.

RIPPEL R. H., HARMON B. G., JENSEN A. H., NORTON H. W., BECKER D. E., 1965a. Response of the gravid gilt to levels of protein as determined by nitrogen balance. J. anim. Sci., 24, 209-215.

RIPPEL R. H., HARMON B. G., JENSEN A. H., NORTON H. W., BECKER D. E., 1965b. Essential amino acid supplementation of intact protein fed to the gravid gilt. J. anim. Sci., 24, 373-377.

ROBINSON D. W., 1969. The cellular response of porcine skeletal muscle to prenatal and neonatal stress. Growth, 33, 231-240.

ROMBAUTS P., 1962. Evolution de l'anabolisme gravidique chez la truie en fonction de l'âge de l'animal. Ann. Zootech., 11, 39-51.

RUIZ M. E., EWAN R. C., SPEER V. C., 1971. Serum metabolites of pregnant and hysterectomized gilts fed two levels of energy. J. anim. Sci., 32, 1153-1159.

SALMON-LEGAGNEUR E., 1963. Influence relative de l'azote el de l'énergie de la ration sur le comportement nutritionnel de la truie gestante : anabolisme gravidique, protéinogenèse, lipogenèse et évolution du contenu utérin. Arch. Sci. physiol., 17, 233-245.

SALMON-LEGAGNEUR E., 1964. Relations entre les graisses ingérées, les lipides corporels et les acides gras du lait chez la truie. Ann. Zootech., 4, 141-155. 
SALMON-LEGAGNEUR E., 1965. Quelques aspects des relations nutritionnelles entre la gestation et la lactation chez la truie. Ann. Zootech., 14, no 1 H. S., 1-137.

SALMON-LEGAGNEUR E., GOMEZ G., JACQUOT R., 1960. Influence de la suralimentation sur la production laitière de la truie. C. R. Acad. Agric. France, 46, 445-451.

SALMON-LEGAGNEUR E., JACQUOT R., 1961a. Modifications corporelles entraînées par l'anabolisme gravidique. C. R. Acad. Sci. Paris, 253, 544-546.

SALMON-LEGAGNEUR E., JACQUOT R., 1961b. Influence du niveau alimentaire sur le comportement nutritionnel de la truie gestante. C. R. Acad. Sci. Paris, 253, 1497-1499.

SEERLEY R. W., PACE T. A., FOLEY C. W., SCARTH R. D., 1974. Effect of energy intake prior to parturition on milk lipids and survival rate, thermostability and carcass composition of piglets. J. anim. Sci., 38, 64-70.

VAN SPAENDONCK R., 1972. Contribution to the study of the energy requirements of sows during gestation. Thèse PP. 252, Univ. Gand.

VERMEDAHL L. D., MEADE R. J., HANKE H. E., RUST J. W., 1969. Effects of energy intake of the dam on reproductive performance, development of offspring and carcass characteristics. J. anim. Sci., 28, 465-472.

VERSTEGEN M. W. A., VAN ES A. J. H., NIJKAMP N. J., 1971. Some aspects of energy metabolism of the sow during pregnancy. Anim. Prod., 13, 677-684.

YOUNG L. G., FORSHAW R. P., SMITH G. C., 1976. Protein supplementation of barley diets for two breeds of gestating swine over two parities. J. anim. Sci., 42, 1182-1186. 